\title{
Síntesis, caracterización y evaluación de recubrimientos híbridos porosos sol-gel dopados con acetato de cerio sobre la aleación de magnesio WE54-AE
}

\section{Synthesis, characterization and evaluation of porous hybrid sol-gel coatings doped with cerium acetate on the WE54-AE magnesium alloy}

\author{
Carlos Andrés Hernández Barrios \\ Ingeniero Químico, estudiante de Doctorado en Ingeniería Mate- \\ riales, Universidad Industrial de Santander, Colombia. \\ carloshernandezbarrios@hotmail.com \\ Leidy Milena Hernández Eugenio \\ Ingeniera Química, egresada \\ Universidad Industrial de Santander, Colombia. \\ leidymile1623@hotmail.com \\ Ana Emilse Coy Echeverría \\ Ph.D. en Ciencia y Tecnología de Materiales, profesora de la \\ Escuela de Ingeniería Metalúrgica y Ciencia de Materiales \\ Universidad Industrial de Santander, Colombia. \\ anaecoy@yahoo.es
}

\author{
Nayda Zuleima Duarte Peñaranda \\ Ingeniera Química, egresada \\ Universidad Industrial de Santander, Colombia. \\ naydazul@hotmail.com \\ Darío Yesid Peña Ballesteros \\ Ph.D. en Corrosión, profesor de la Escuela de Ingeniería \\ Metalúrgica y Ciencia de Materiales \\ Universidad Industrial de Santander,Colombia. \\ darioypb@gmail.com \\ Fernando Viejo Abrante \\ Ph.D. en Ciencia y Tecnología de Materiales, profesor de la \\ Escuela de Ingeniería Química \\ Universidad Industrial de Santander, Colombia. \\ fviejo@hotmail.com
}

\begin{abstract}
Resumen-El objetivo del presente trabajo fue sintetizar y caracterizar recubrimientos híbridos sol-gel base sílice dopados con inhibidores de corrosión sobre la aleación de magnesio WE54-AE. Se establecieron como variables de síntesis el tiempo de envejecimiento (hasta 24 horas) y la proporción del agente de entrecruzamiento 3- aminopropiltrietoxisilano, APTES. El inhibidor de corrosión seleccionado fue acetato de cerio en contenido $0,5 \%$ molar. El proceso de gelificación fue monitoreado mediante espectroscopía de infrarrojo con transformada de Fourier (FT-IR) y medidas de viscosidad, mientras que los recubrimientos fueron caracterizados mediante microscopía electrónica de barrido (MEB-EDX). Por otro lado, la resistencia a la corrosión fue evaluada a través de ensayos electroquímicos en solución $0,1 \mathrm{M}$ de $\mathrm{NaCl}$. Los resultados obtenidos confirmaron que, en general, los recubrimientos obtenidos presentan una mejora de la resistencia a la corrosión con relación a la aleación base. Así mismo, se observó que las variables estudiadas afectaron sustancialmente las características protectoras del recubrimiento, de tal forma que, bajo determinadas condiciones, es posible obtener recubrimientos altamente homogéneos y porosos, característica necesaria para albergar inhibidores de corrosión.
\end{abstract}

Palabras clave— Magnesio, Corrosión, Recubrimientos, Sol-gel.

\begin{abstract}
The aim of the present work was the synthesis and characterization of silica-based hybrids coatings sol-gel doped with corrosion inhibitors (cerium acetate, $0.5 \%$ molar) on the WE54-AE magnesium alloy. The ageing time and the proportion of the crosslinking agent (3-aminopropyltrimethoxysilane, APTES) were modified to evaluate their influence on the sol-gel processing and the features of the coatings obtained. The sol evolution was monitored by infrared spectroscopy (FTIR) and viscosity measurements, while the sol-gel coatings were characterized by scanning electron microscopy (SEM-EDX). Further, corrosion resistance was evaluated by electrochemical testing in $0,1 \mathrm{M} \mathrm{NaCl}$ solution. The results obtained showed that, in general, the sol-gel coatings were highly stable and provided an enhancement in corrosion resistance when compared to the uncoated alloy. In addition, the morphology of the coating resultant was substantially affected by the modification of either the ageing time or the APTES proportion. In this regard, under specific conditions, it was possible to obtain highly homogeneous and porous films, which are adequate to accommodate corrosion inhibitors.
\end{abstract}

Keywords- Magnesium, Corrosion, Coatings, Sol-gel. 


\section{INTRODUCCIÓN}

En la actualidad, el magnesio es el material estructural más liviano que se usa en ingeniería, con una densidad de $1,74 \mathrm{~g} / \mathrm{cm}^{3}$, es $35 \%$ más ligero que el aluminio $\left(2,7 \mathrm{~g} / \mathrm{cm}^{3}\right)$ y cuatro veces menos pesado que el acero $\left(7,86 \mathrm{~g} / \mathrm{cm}^{3}\right)$, siendo su resistencia específica mayor que la de otros materiales [1]-[4]. La elevada resistencia específica del magnesio hace que sus aleaciones sean adecuadas para aplicaciones donde la reducción de peso es importante, fundamentalmente en la fabricación de componentes dentro de la industria automotriz y aeronáutica, con objeto de minimizar el consumo de combustible y, por tanto, las emisiones de $\mathrm{CO}_{2}$

En los últimos años se han desarrollado diferentes aleaciones de magnesio entre las que se encuentran las aleaciones WEXX (Mg-Y-RE), donde la adición de itrio y tierras raras (RE, RareEarths) mejora las propiedades mecánicas y la resistencia a diferentes temperaturas. Por esta razón, dichas aleaciones encuentran principal aplicación en la industria del transporte en la fabricación de piezas de responsabilidad moderada, como partes de los chasis de automóviles, fuselajes de trenes, tranvías, aviones, etc. [5]-[7].

Sin embargo, en la actualidad el número de posibles aplicaciones de las aleaciones de magnesio es muy bajo y sus excelentes prestaciones mecánicas son usualmente desaprovechadas, debido fundamentalmente a su baja resistencia a la corrosión, que las hace vulnerables en la mayoría de los medios a los que son sometidas [1], [8]. Estas aleaciones pueden presentar diversos tipos de mecanismos de corrosión como corrosión localizada, galvánica, intergranular, por fatiga, entre otras. En este sentido, la resistencia a la corrosión de las aleaciones de magnesio se ve notoriamente reducida cuando contiene impurezas metálicas de $\mathrm{Fe}, \mathrm{Ni}$ o $\mathrm{Cu}$ por encima del límite de tolerancia y por la existencia de fases secundarias como $\mathrm{Mg}_{17} \mathrm{Al}_{12}, \mathrm{Al}_{8} \mathrm{Mn}_{5}, \mathrm{Mg}_{12} \mathrm{Nd}$, entre otras, asociada a la presencia de elementos aleantes [9], [10].

Para minimizar este problema y aumentar en gran medida la aplicación de las aleaciones de magnesio a nivel industrial, se han desarrollado diversos mecanismos de protección de su superficie metálica para hacerla químicamente resistente a los medios en los que pueden estar ex- puestas. Dentro de los principales tratamientos de protección se destaca la tecnología sol-gel, método ampliamente utilizado para la producción de películas de naturaleza vítrea o cerámica, que ofrecen una barrera físico-química que protege al metal del medio corrosivo. Entre sus principales ventajas se encuentran la bajas temperaturas de procesamiento; la capacidad de obtener películas superficiales o recubrimientos con propiedades específicas deseadas, y que pueden ser elegidas de manera aleatoria, desde recubrimientos de elevada superficie específica y porosidad, hasta recubrimientos densos y compactos [11], [12]; la capacidad de producir recubrimientos en piezas con formas complejas; y el uso de procedimientos simples de aplicación fácilmente adaptables a escala industrial, tales como la técnica "dipcoating".

Dentro de la síntesis sol-gel se han venido utilizando dos tipos de precursores: de películas inorgánicas y orgánicas. Los precursores de películas inorgánicas son empleados con objeto de obtener recubrimientos cerámicos base $\mathrm{SiO}_{2}, \mathrm{ZrO}_{2}, \mathrm{TiO}_{2}$ cuyas características fundamentales son su elevada adhesión y resistencia al desgaste [13]. Sin embargo, han presentado restricciones en su aplicación por el límite de espesor de la película y la formación de grietas debido a las tensiones que se originan durante el secado y el tratamiento térmico [2], [12], [13]. Estos precursores suelen ser tipo metal-alcóxido, M (OR)n donde $M$ puede ser $\mathrm{Si}, \mathrm{Ti}, \mathrm{Zr}, \mathrm{Al}, \mathrm{Fe}$ o B, mientras que $\mathrm{R}$ es un grupo alquilo. De este modo, ya han sido utilizados de forma extensiva para la formación de recubrimientos sol-gel, precursores como el tetraetoxisilano (TEOS), el tetrametoxisilano (TMOS), o propóxidos de zirconio o titanio, entre otros. No obstante, la mayoría de los precursores inorgánicos suelen ser base silano (Si (OR)n) por su reactividad moderada, que hace más controlable el proceso de síntesis.

Por otro lado, los precursores de películas orgánicas suelen emplearse para aumentar la flexibilidad y la porosidad, lo que permite obtener películas más flexibles y con menos tendencia al agrietamiento, además de disminuir las temperaturas y los tiempos de curado [13]. Este tipo de precursores suelen ser organoalcoxisilanos de estructura Ro-Si $(\mathrm{OR})_{3}$, donde Ro es un grupo funcional orgánico tipo amino, epoxy, vinil, metacrila- 
to, entre otros. El precursor glicidiltrimetoxisilano (GPTMS) es uno de los más usados para aumentar la resistencia a la corrosión de los recubrimientos sol-gel, porque origina estructuras aislantes y de elevada densidad debido a la presencia del grupo epoxy. Así mismo, el precursor 3-aminopropiltrimetoxisilano (APTES) es ampliamente usado como acelerador del proceso de hidrólisis-condensación y agente de entrecruzamiento, ya que la presencia del grupo amino en su estructura genera un ataque nucleófilo sobre los grupos epoxi presentes en las soluciones sol-gel, formando redes tridimensionales base sílice e incrementando a su vez la viscosidad y el pH de los soles, generando que el proceso se desarrolle en un ambiente básico. Este hecho hace que la estructura final del recubrimiento sea más entrecruzada que cuando se sintetiza a partir de soles en medio ácido; sin embargo, estas características se desarrollan de una manera agresiva, ya que el aumento del $\mathrm{pH}$ y la viscosidad son realmente considerables en tiempos muy cortos, lo que puede llegar a generar un entrecruzamiento acelerado que propicia la formación de estructuras con mayor grado de porosidad [14], [15].

Actualmente, se está investigando la posibilidad de implementar una combinación de los dos precursores (inorgánico-orgánico) para la producción de recubrimientos híbridos. Este método busca hacer uso de la gran adhesión de los recubrimientos inorgánicos y de la baja tendencia al agrietamiento de los orgánicos, para la obtención de películas con mejores propiedades mecánicas y mayor resistencia a la corrosión [14], [16], [17]. Así mismo, mediante un control adecuado del $\mathrm{pH}$ del sol se pueden crear estructuras porosas, fundamentalmente en presencia del precursor APTES, que permiten la posibilidad de incluir inhibidores de corrosión que actúan como dopantes y que otorgan al recubrimiento propiedades autocurativas en caso de que exista un daño bajo condiciones de operación. Entre los inhibidores más utilizados se encuentran las sales de tierras raras, fundamentalmente de cerio, ya que este elemento forma hidróxidos insolubles que bloquean las áreas activas y detienen la formación de pares galvánicos con la matriz metálica, desacelerando el proceso de corrosión [18].

Con base en las anteriores premisas, el objetivo del presente proyecto fue sintetizar, caracte- rizar y evaluar recubrimientos híbridos porosos sol-gel base TEOS-GPTMS-APTES dopados con sales de cerio, depositados sobre la aleación de magnesio WE54-AE, estudiando específicamente el tiempo de envejecimiento y la proporción del precursor orgánico APTES, variables que afectan directamente al proceso de síntesis, las características morfológicas y la resistencia a la corrosión de dichos recubrimientos.

\section{PROCEDIMIENTO EXPERIMENTAL}

\subsection{Material objeto de estudio}

El material objeto de estudio fue la aleación de magnesio WE54-AE suministrada por MagnesiumElektron (Reino Unido), cuya composición química nominal de acuerdo con la información del proveedor es: Nd: 1,5-2,0\%; Y: 4,8-5,5\%; RE: 1,02,0\%; Zr: 0,4\% y Mg: balance (\% en peso).

\subsection{Caracterización microestructural del material objeto de estudio}

Se prepararon superficialmente muestras de la aleación mediante un proceso de desbaste y pulido. Para el desbaste se utilizó papel abrasivo hasta granulometría P1200, y para el pulido, pasta de diamante de 3 y $1 \mu \mathrm{m}$ hasta obtener un acabado superficial tipo especular; a continuación, las muestras fueron lavadas con etanol y secadas con una corriente de aire caliente. Finalmente, con el fin de revelar la microestructura general del material, las muestras fueron atacadas con solución de ácido pícrico (10ml de ácido acético y $4.2 \mathrm{~g}$ de ácido pícrico en $70 \mathrm{ml}$ de etanol), según la norma ASTM E-407 "Standard Practicefor Microetching Metals and Alloys" [19]. La caracterización microestructural se realizó mediante el microscopio óptico OLYMPUS GX71.

\subsection{Preparación de recubrimientos sol-gel híbridos}

\subsubsection{Preparación de los soles híbridos}

Con objeto de obtener soles que dieran lugar a recubrimientos de naturaleza porosa se diseñó una ruta de síntesis en dos etapas: envejecimiento en medio ácido y envejecimiento en medio básico. Inicialmente, se llevó a cabo el proceso de 
hidrólisis y condensación (envejecimiento) en medio ácido $(\mathrm{pH}=5)$ de los precursores TEOS y GPTMS que fueron mezclados a temperatura ambiente en una proporción molar constante de 3:1 usando etanol como solvente y con una agitación constante. Como agente ácido se utilizó ácido acético diluido $(0,5 \% \mathrm{vol})$ que se agregó en proporción volumétrica $1: 1$ con respecto a la mezcla TEOS-GPTMS. Con el fin de evaluar el efecto del tiempo de envejecimiento en la calidad de los soles y recubrimientos, se establecieron tiempos de estudio entre 15 minutos y 24 horas. Este tiempo de envejecimiento fue establecido entre el momento en que fue agregado el ácido acético a la mezcla de precursores (TEOS y GPTMS) y el instante en que se añadió el agente de entrecruzamiento (APTES). En la segunda etapa del proceso, se agregó el precursor orgánico APTES que actuó de agente de entrecruzamiento y garantizó condiciones básicas $(\mathrm{pH}=9-10)$ de envejecimiento. Dicho precursor, debido a su elevada reactividad, fue añadido lentamente para permitir que la gelificación de los soles fuera homogénea. Por otro lado, con el fin de evaluar su influencia en el proceso solgel, se fijaron las siguientes proporciones molares: 0,05:1, 0,15:1 y 0,25:1 de APTES respecto al precursor GPTMS. Para estos soles se fijó un tiempo de 30 minutos de envejecimiento básico adicionales, posterior a la adición del APTES, antes de llevar a cabo la deposición del sol. Con objeto de estudiar el efecto del cerio, los recubrimientos más representativos en términos de morfología y resistencia a la corrosión, fueron dopados con una proporción 0,5\% molar de acetato de cerio $\left[\mathrm{Ce}\left(\mathrm{C}_{2} \mathrm{H}_{3} \mathrm{O}_{2}\right)_{3} \cdot 1.5 \mathrm{H}_{2} \mathrm{O}\right]$.

\subsubsection{Aplicación de recubrimientos mediante dip-coating}

Previo al proceso de deposición de los recubrimientos, muestras de cada aleación fueron desbastadas con papel abrasivo hasta granulometría 600 y, posteriormente, secadas con etanol y aire caliente. La deposición del sol sobre la superficie metálica se realizó mediante dip-coating. Las muestras fueron sumergidas en soles con distintos tiempos de envejecimiento, a una velocidad de inmersión constante de $6 \mathrm{~cm} / \mathrm{min}$, donde permanecieron sumergidas por 2 minutos para garantizar la mojabilidad y adhesión del recubrimiento sobre la superficie de la aleación [18]. Cumplido dicho tiempo, las muestras fueron retiradas conservando la misma velocidad utilizada en el proceso de inmersión.

\subsubsection{Tratamiento térmico de secado}

Después de la obtención de los recubrimientos, las muestras fueron expuestas al aire libre durante 30 minutos para favorecer la evaporación masiva del disolvente (etanol). A continuación, se realizó un tratamiento térmico de secado-curado en estufa, compuesto por dos etapas: una primera etapa a una temperatura de $60^{\circ} \mathrm{C}$ por $4 \mathrm{~h}$ seguida de una posterior a $120^{\circ} \mathrm{C}$ por $2 \mathrm{~h}$. Este tratamiento se realizó para garantizar la evaporación completa del solvente y el agua contenidos en la película, así como para evitar la formación de grietas.

\subsection{Caracterización de los soles híbridos}

\subsubsection{Espectroscopía Infrarroja}

Con objeto de estudiar las reacciones de hidrólisis y condensación de los precursores durante el proceso de gelificación, se llevó a cabo un análisis mediante espectroscopía infrarroja de los soles antes y después de agregar el precursor APTES. Para ello, se utilizó el espectrofotómetro infrarrojo con transformada de Fourier (FTIR) SHIMADZU modelo 84005.

\subsubsection{Análisis Reológico}

De forma complementaria, para de evaluar el efecto de la viscosidad del sol en la calidad final de los recubrimientos, se realizó un estudio reológico del proceso de gelificación. Para ello se utilizó el reómetro digital BROOKFIELD modelo DV.III + RHEOMETER V5 con una aguja ULAO.0. Los datos de viscosidad (CP) fueron registrados cada $5 \mathrm{mi}-$ nutos durante un tiempo máximo de 24 horas a una temperatura de $25^{\circ} \mathrm{C}$ aproximadamente.

\subsection{Caracterización de los recubrimientos sol- gel híbridos}

La caracterización de los recubrimientos se llevó a cabo utilizando un microscopio electrónico de barrido JEOL JSM 6490, equipado con detectores para el análisis de energías dispersivas de rayos $X$ 
(EDX) y para la obtención de imágenes por electrones retrodispersados (BSE).

\subsection{Evaluación de la resistencia a la corrosión de los recubrimientos}

La protección frente al fenómeno de corrosión ofrecida por los recubrimientos fue evaluada mediante ensayos electroquímicos de polarización anódica potenciodinámica realizados con el potenciostato ACM Instruments. Se utilizó una celda de tres electrodos, con un electrodo de referencia (calomelanos), un electrodo auxiliar (platino) y un electrodo de trabajo (muestra). Los ensayos se realizaron en una solución 0,1M de $\mathrm{NaCl}$. Las muestras, con un área aproximada de $1 \mathrm{~cm}^{2}$, se sumergieron en la solución salina por 30 minutos con objeto de permitir la estabilización del sistema. Una vez alcanzado dicho tiempo, se procedió a realizar el ensayo de polarización anódica en el que se llevó a cabo un barrido anódico de potencial desde $-100 \mathrm{mV}$ hasta $2000 \mathrm{mV}$ (con respecto al potencial de circuito abierto (OCP)), con una velocidad de barrido de $0,3 \mathrm{mV} / \mathrm{s}$ y una densidad de corriente límite de $1 \mathrm{~mA} / \mathrm{cm}^{2}$.

\section{RESULTADOS}

\subsection{Caracterización microestructural de los materiales objeto de estudio}

En la Fig. 1 se presentan las micrografías ópticas de la aleación WE54-AE donde se observa su microestructura típica de extrusión, con granos equiaxiales de $\alpha$-Mg homogéneos, Fig. 1(a), y con presencia de inclusiones intermetálicas de forma irregular, dispersados al azar, tanto dentro como en los bordes de grano, Fig. 1(b). Dichas inclusiones, son específicamente precipitados de itrio, zirconio y tierras raras (RE, RareEarths) de composición $\mathrm{Mg}_{14} \mathrm{YNd}_{2}$ [5], [20].

\subsection{Caracterización de los soles}

\subsubsection{Espectroscopía FTIR}

En la Fig. 2(a) se presentan los espectros FTIR de los soles a diferentes tiempos en ausencia del agente de entrecruzamiento APTES. Se observa que a medida que se incrementa el tiempo de envejecimiento en medio ácido, comienza a reve- larse una nueva banda en $1050 \mathrm{~cm}^{-1}$ que se encuentra relacionada con la tensión originada por los enlaces $\mathrm{Si}-\mathrm{O}-\mathrm{Si}$, indicando que las reacciones de hidrólisis y condensación de las moléculas de los precursores se han iniciado. Esta banda se advierte con mayor intensidad al cabo de 24 h. Sin embargo, sigue apareciendo la banda de 1080 $\mathrm{cm}^{-1}$, hecho indicador de que el proceso de hidrólisis no se ha llevado a cabo de forma completa.

Fig. 1. MICROGRAFÍA ÓPTICA DE LA ALEACIÓN WE54-AE REVELADA CON SOLUCIÓN DE ÁCIDO PÍCRICO A) VISTA GENERAL, B) DETALLE
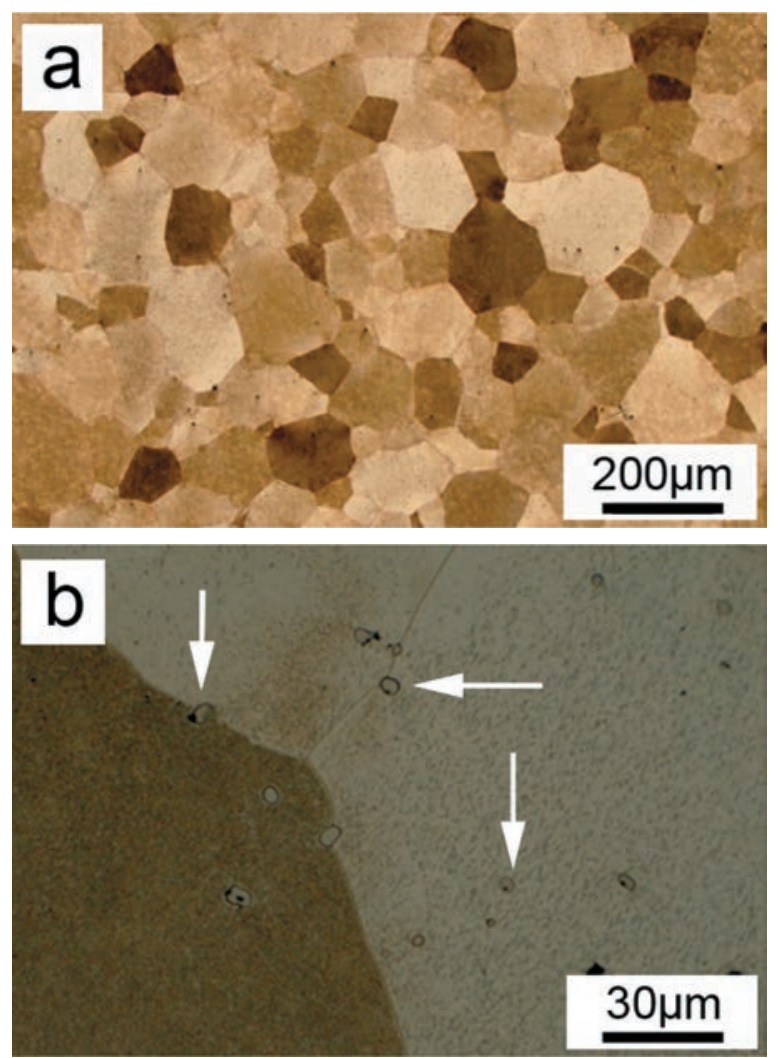

Para evaluar el efecto del agente de entrecruzamiento APTES en el proceso de envejecimiento, se estudiaron soles a diferentes tiempos después de agregar dicho precursor (envejecimiento en medio básico). Los espectros infrarrojos de estos soles se presentan en la Fig. 2(b). Se observa que para tiempos cortos después de añadido el agente de entrecruzamiento, las bandas principales asociadas a los enlaces Si-O-C (1080 $\mathrm{cm}^{-1}$ ) y Si-OH desaparecen completamente y que la banda asociada al enlace Si-O-Si $\left(1050 \mathrm{~cm}^{-1}\right)$ aumenta notablemente su intensidad. Este hecho parece indicar que el precursor APTES, acelera considerablemente el proceso de hidrólisiscondensación. 
Fig. 2. ESPECTROS FTIR DE LOS SOLES: A) ANTES Y B) DESPUÉS DE AGREGAR EL PRECURSOR APTES
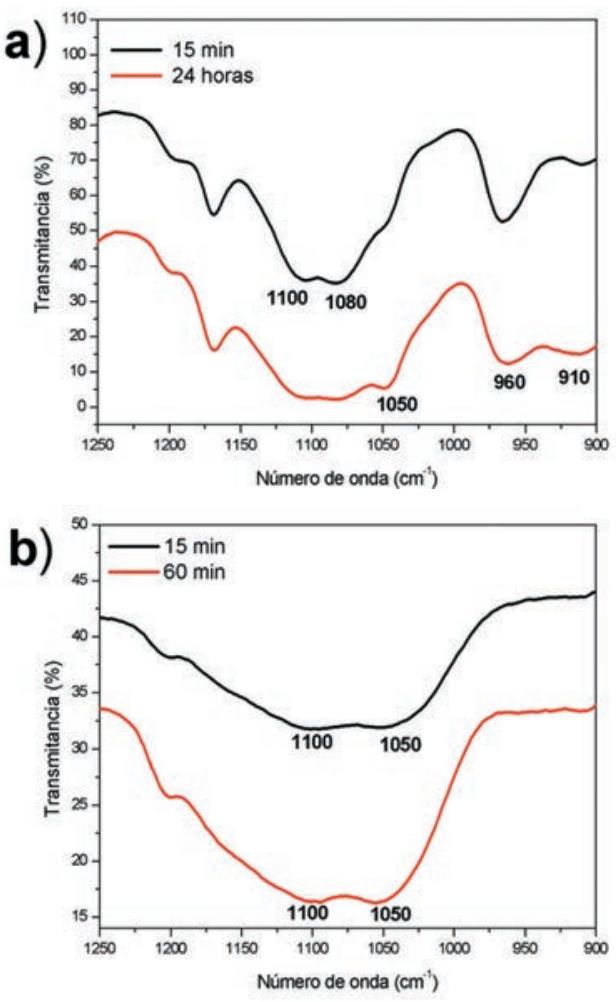

\subsubsection{Caracterización reológica de los soles}

En la Fig. 3 se presentan las curvas viscosidad respecto al tiempo para soles preparados con diferentes proporciones molares de APTES/GPTMS. Con respecto al sol base se observó un cambio de $4 c P$ después de 24 horas.Por otro lado, para soles en presencia de APTES, la viscosidad de los soles aumentó con el tiempo siguiendo un crecimiento exponencial hasta el momento en el cual el sol se gelificó completamente. Es significativo el hecho de que pequeños incrementos en la proporción de APTES en el sol modificaron de forma notoria la viscosidad, acelerando el proceso de gelificación. Ejemplo de ello fue evidenciado en los soles preparados con proporciones 0,25:1 para los que se observó que, en el periodo comprendido entre 30 a 50 min, la viscosidad del sol se incrementó alrededor de un orden de magnitud pasando de valores de 3,5 a $32 \mathrm{cP}$, valor final en el que el sol estaba completamente gelificado. Este hecho está asociado a que la adición de APTES ocasionó que el proceso de hidrólisis-condensación se de- sarrollara en medio básico e incrementó la velocidad de gelificación del sol [14], [15].

Fig. 3. EVOLUCIÓN DE LA VISCOSIDAD DE DISTINTOS SOLES PREPARADOS CON DIFERENTES PROPORCIONES MOLARES DE APTES: GPTMS

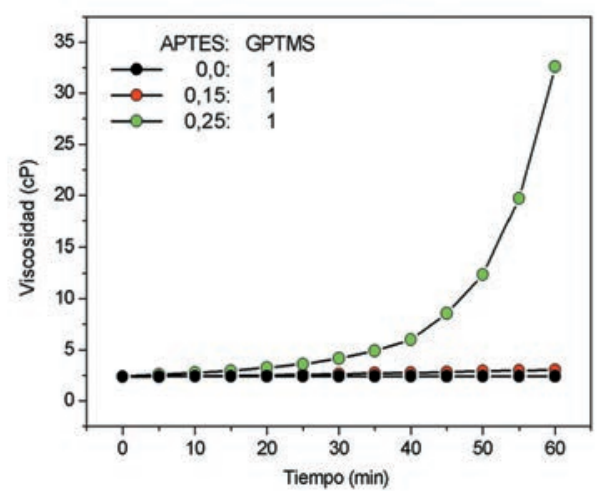

\subsection{Caracterización de los recubrimientos sol- gel híbridos}

En la Fig. 4 se muestran la micrografías electrónicas del recubrimiento realizado sobre la aleación WE54-AE, para un tiempo de 24h, en ausencia del precursor APTES. Se observa que, bajo condiciones de envejecimiento ácidas, los recubrimientos obtenidos presentan buenas características morfológicas y ausencia de porosidad, Fig. 4(a). El menor valor de $\mathrm{pH}$ de los soles sin APTES hace que el proceso de gelificación sea más lento y controlado, y permite además una evaporación más uniforme del solvente. Sin embargo, las partículas de itrio presentes en la aleación hacen que el recubrimiento no se adhiera completamente y forme grietas locales alrededor de los sitios donde estas partículas están presentes, Fig. 4(b).

Por otro lado, los recubrimientos realizados en presencia del precursor APTES se muestran en la Fig. 5. De manera general, para los soles con altas proporciones de APTES: GPTMS y bajos tiempos de envejecimiento en medio ácido, el proceso de gelificación fue demasiado violento, generando recubrimientos poco continuos, porosos y presencia de grietas en la superficie, Fig. 5(a). Lo anterior está relacionado con la elevada velocidad de gelificación que impide la evaporación controlada del solvente (etanol y agua) que es atrapado dentro de la red del gel en grandes proporciones, y que durante la etapa de secado posterior da lugar a estructuras porosas de baja uniformidad. Sin embargo, un aumento del tiempo de envejecimiento en medio ácido previo a la adición de APTES favorece un pro- 
ceso de hidrólisis y condensación, así como una evaporación de solvente, más uniformes, y permiten obtener recubrimientos continuos con una porosidad homogénea y controlada, Fig. 5(b).

\subsection{Evaluación de la resistencia a la corrosión de los recubrimientos}

\subsubsection{Ensayo de polarización anódica potenciodinámica}

En las curvas de polarización anódica mostradas en la Fig. 6 se puede apreciar el efecto de la adición de APTES en las características protectoras del recubrimiento para tiempos de envejecimiento en medio ácido de 15 minutos y 6 horas. En primer lugar, se observa que la aleación base (sin recubrir) al ser sumergida en la solución de ensayo, presenta una resistencia a la corrosión relativamente baja, con tendencia a sufrir corrosión por picadura, Fig. 6(a). Interesantemente, se observan efectos contrapuestos con relación a la proporción de APTES y el tiempo de envejecimiento en medio ácido. En este sentido, para tiempos cortos de envejecimiento, a medida que aumenta la relación molar APTES: GPTMS, la resistencia a la corrosión del recubrimiento se hace cada vez mayor, al disminuir la densidad de corriente de corrosión y aumentar el rango de pasivación. Por el contrario, para tiempos prolongados de envejecimiento, el aumento de la relación molar APTES: GPTMS en los soles, generó recubrimientos con escasas características protectoras, Fig. 6(b).

Con el fin de estudiar el efecto del aumento del tiempo de envejecimiento en la resistencia a la corrosión de los recubrimientos, en la Fig. 7 se presentan las curvas de polarización de las películas a diferentes tiempos de envejecimientos y proporciones molares APTES: GPTMS de 0,00:1 y 0,25:1.

En ausencia de APTES, los recubrimientos presentan una ligera mejoría en la resistencia a la corrosión, con una disminución de la densidad de corriente de corrosión, Fig. 7(a). No obstante, no se aprecia un efecto significativo del tiempo de envejecimiento en medio ácido en las características protectoras del mismo. Por otro lado, para elevadas concentraciones de APTES, un aumento del tiempo de envejecimiento da lugar a recubri- mientos cada vez menos protectores con una resistencia incluso inferior que la aleación base, Fig. 7 (b). La mayor o menor resistencia a la corrosión posiblemente está asociada al menor o mayor grado de porosidad de los recubrimientos.

En la Tabla I se listan los parámetros electroquímicos más importantes de las curvas de polarización de la Fig. 6, hallados según la norma ASTM G3-89 "Standard Practicefor Conventions Applicable to Electrochemical Measurements in CorrosionTesting" [21].

Con el objetivo de evaluar la influencia del acetato de cerio en la resistencia a la corrosión, se escogieron los recubrimientos con condiciones de 0,05:1 - 6h y 0,25:1-15 min, debido a que eran los más representativos en cuanto a características morfológicas y protectoras. En este sentido, en la Fig. 8 se presentan las curvas de polarización de la aleación WE54-AE recubierta con películas sol-gel dopadas con acetato de cerio ( $0,5 \%$ molar), bajo dichas condiciones en comparación con el recubrimiento en ausencia de inhibidor de corrosión.

Fig. 4.MICROGRAFÍAS ELECTRÓNICAS BSE DEL RECUBRIMIENTO OBTENIDO SOBRE LA ALEACIÓN WE54-AE PARA UN TIEMPO DE 24H ANTES DE AGREGAR EL PRECURSOR APTES
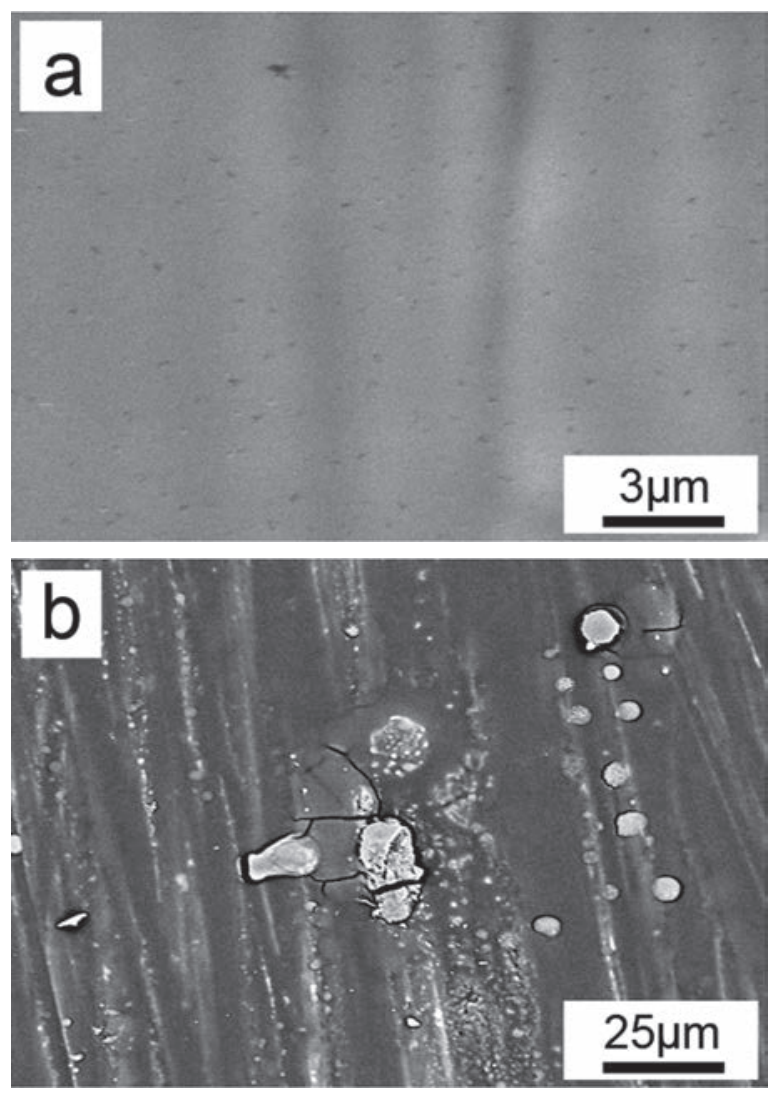
Fig. 5. MICROGRAFÍAS ELECTRÓNICAS DE LOS RECUBRIMIENTOS DEPOSITADOS SOBRE LA ALEACIÓN WE54 CON PROPORCIÓN APTES:GPTMS DE 0,25:1 Y TIEMPO DE ENVEJECIMIENTO EN MEDIO ÁCIDO DE: A) 15 MIN Y B) 6 HORAS
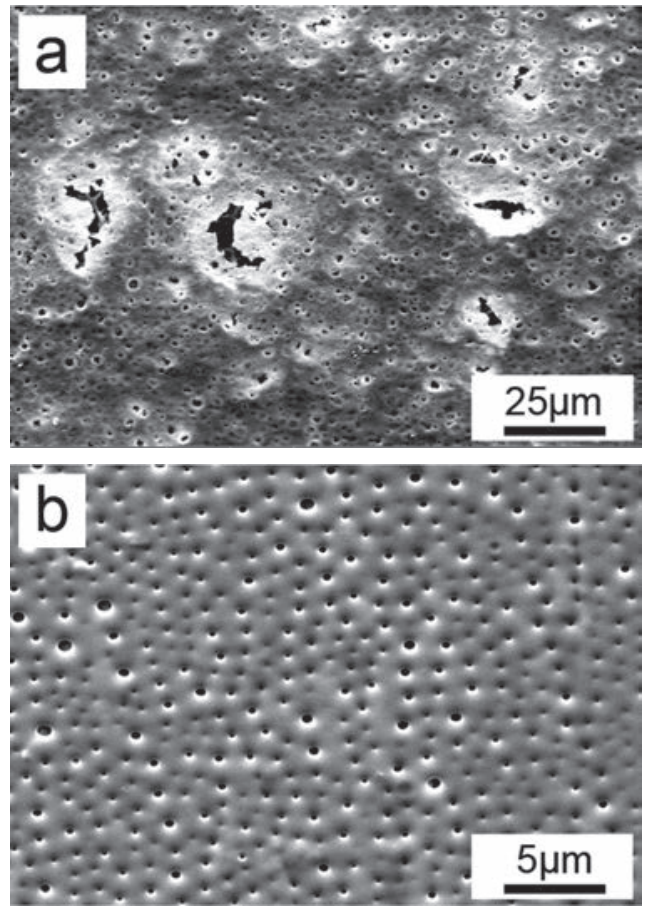

Fig. 6. CURVAS DE POLARIZACIÓN ANÓDICA DEL MATERIAL BASE Y RECUBIERTO A PARTIR DE SOLES CON DIFERENTES PROPORCIONES MOLARES DE APTES: GPTMS Y TIEMPOS DE ENVEJECIMIENTO DE: A) 15 MIN Y B) 6 HORAS
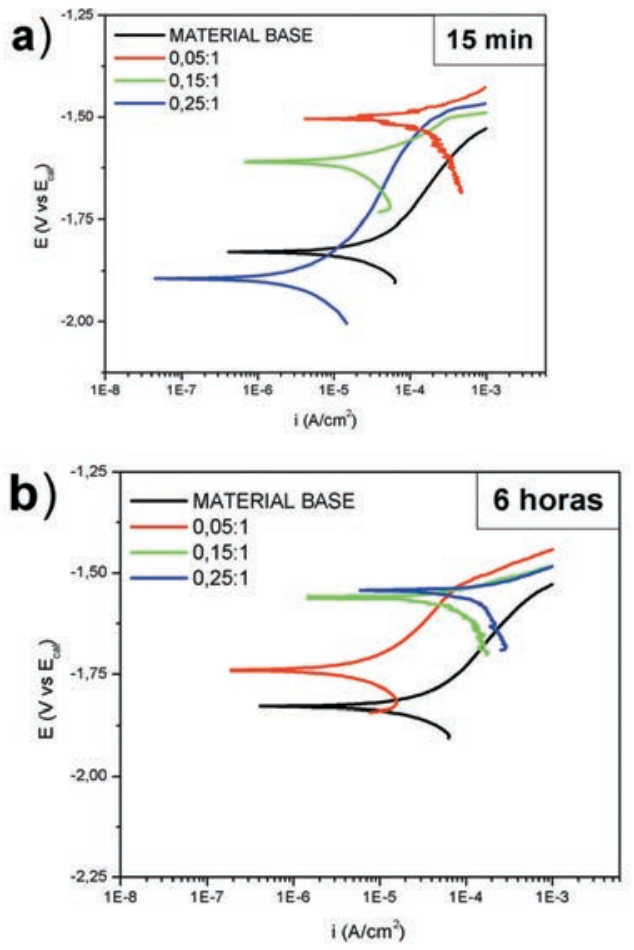

Fig. 7. CURVAS DE POLARIZACIÓN ANÓDICA DEL MATERIAL BASE Y RECUBIERTO A PARTIR DE SOLES CON PROPORCIONES MOLARES APTES: GPTMS DE: A) 0,00:1 Y B) 0,25:1; A DIFERENTES TIEMPOS DE ENVEJECIMIENTO
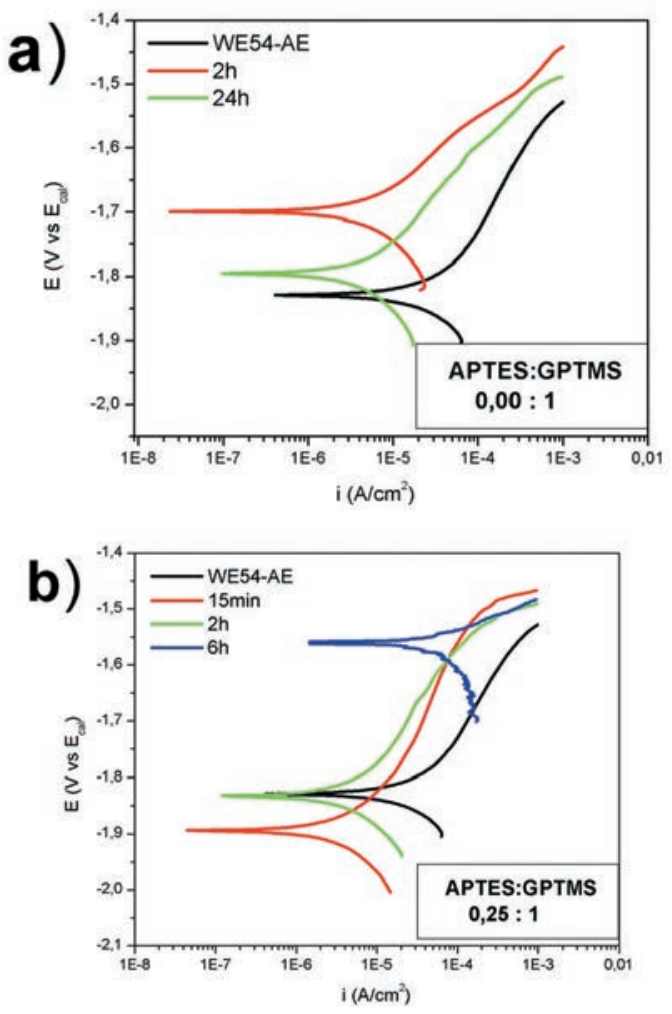

TABLA I

PARÁMETROS ELECTROQUÍMICOS EXTRAÍDOS DE LAS CURVAS DE POLARIZACIÓN DE LA FIG. 6.

\begin{tabular}{|c|c|c|c|c|}
\hline MATERIAL & $E_{\text {corr }}(m V)$ & $E_{p i c}(m V)$ & $\begin{array}{c}\mathbf{i}_{\text {corr }} \\
\left(\mathrm{A} / \mathrm{cm}^{2}\right)\end{array}$ & $\mathrm{E}_{\mathrm{pic}}-\mathrm{E}_{\mathrm{corr}}(\mathrm{mV})$ \\
\hline Aleación Base & -1823 & - & $1,90 \mathrm{E}-5$ & - \\
\hline $\begin{array}{l}\text { Recubrimiento } \\
\text { APTES:GPTMS - t }\end{array}$ & & & & \\
\hline $0,05: 1-15 \mathrm{~min}$ & -1506 & - & $1,59 E-4$ & - \\
\hline $0,15: 1-15 \mathrm{~min}$ & -1613 & -1502 & $1,70 \mathrm{E}-5$ & 111 \\
\hline $0,25: 1-15 \mathrm{~min}$ & -1891 & -1504 & $3,0 E-6$ & 387 \\
\hline $0,05: 1-6 h$ & -1741 & -1527 & $7,0 E-6$ & 214 \\
\hline $0,15: 1-6 h$ & -1561 & - & $6,0 E-5$ & - \\
\hline $0,25: 1-6 h$ & -1545 & - & $1,37 E-4$ & - \\
\hline
\end{tabular}


Fig. 8. CURVAS DE POLARIZACIÓN DE LA ALEACIÓN WE54-AE RECUBIERTA CON PELÍCULAS SOL-GEL DOPADAS CON 0,5\% MOLAR DE ACETATO DE CERIO, PARA LAS CONDICIONES: A) 0,05:1-6H Y B) 0,25:1-15MIN
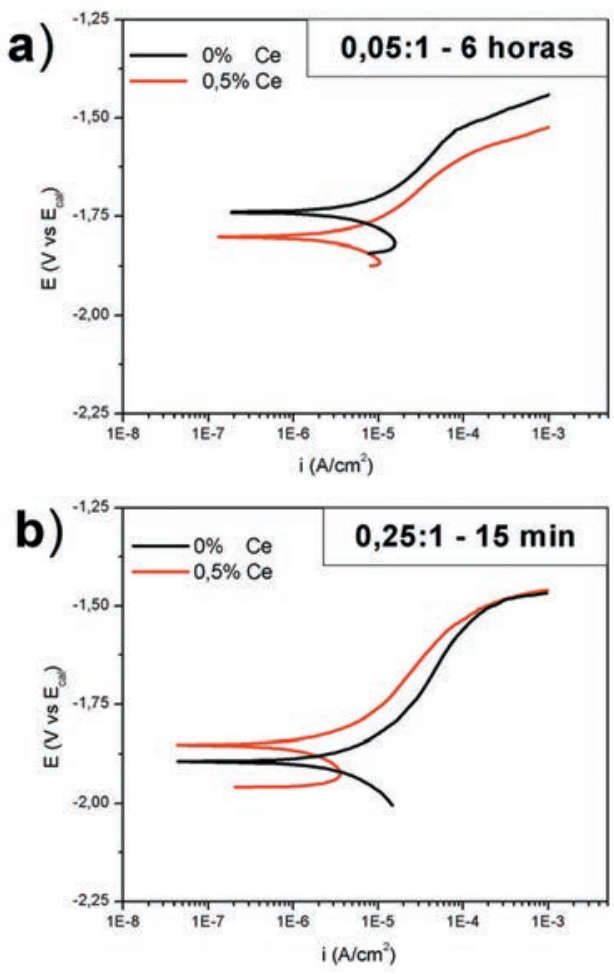

Al comparar las curvas de polarización de los recubrimientos con y sin acetato de cerio, se concluye que después de 30 minutos de inmersión en el medio de ensayo todavía no se alcanza a observar el efecto benéfico de la incorporación del inhibidor en la estructura de las películas. Es evidente que la adición de cerio no modifica significativamente la densidad de corriente de corrosión y el rango de pasivación. No obstante, cabe mencionar que el efecto de la adición de cerio realmente debe ser observado una vez el recubrimiento ha perdido sus características protectoras. Por esta razón, es necesario realizar ensayos electroquímicos tales como espectroscopia de impedancia electroquímica y ensayos de inmersión por tiempos más prolongados, que permitan observar con mayor claridad el fenómeno de autocurado de estos recubrimientos. Esta investigación se está llevando a cabo en la actualidad y se espera que los resultados obtenidos sean mostrados en posteriores publicaciones.

\section{CONCLUSIONES}

Mediante un control adecuado del tiempo de envejecimiento en medio ácido y la proporción del precursor APTES es posible crear recubrimientos sol-gel uniformes, de porosidad controlada, y que pueden permitir albergar inhibidores de corrosión. En este sentido, un aumento del tiempo de envejecimiento favoreció la formación de recubrimientos uniformes y homogéneos, mientras que el incremento de la proporción de APTES aumentó el grado de porosidad de los mismos.

Con relación al efecto de ambas variables en la resistencia a la corrosión, se observó efectos contrapuestos de tal forma que, para tiempos cortos de envejecimiento, a medida que aumenta la relación molar APTES: GPTMS, la resistencia a la corrosión del recubrimiento se hace cada vez mayor; mientras que, por el contrario, para tiempos prolongados de envejecimiento, el aumento de la relación molar APTES: GPTMS en los soles, generó recubrimientos con escasas características protectoras.

Los ensayos de polarización anódica de los recubrimientos dopados con 0,5\% molar de Ce no revelaron un aumento considerable de la resistencia a la corrosión, por lo que es necesario realizar ensayos electroquímicos de larga duración como impedancia electroquímica, con el fin de evaluar el efecto inhibidor del cerio en la estructura de los recubrimientos.

\section{AGRADECIMIENTOS}

Los autores agradecen a la Vicerrectoría de Investigación y Extensión de la Universidad Industrial de Santander (Colombia) por la financiación de este proyecto (Desarrollo de Materiales y Recubrimientos de Interés Tecnológico, Código 5450).

\section{REFERENCIAS}

[1] M. Kulekci, "Magnesium and its alloys applications in automotive industry," Int. J. Adv. Manuf. Tech., vol. 39, no.9-10, pp. 851-865, Nov.2008.

[2] J.E Gray and B. Luan, "Protective coatings on magnesium and its alloys - a critical review," J. Alloys Compd., vol. 336, no.1-2, pp.88-113, Apr. 2002.

[3] D. Mike, "Magnesium Alloys," in Environmental Effects on Engineered Materials, Nueva York: Marcel Dekker Inc, 2001.

[4] A.L.K Tana, et al, "Multilayer sol-gel coatings for corrosion protection of magnesium," Surf. Coat. Technol., vol. 198, no.1-3, pp.478-482, Aug. 2005. 
[5] A.E. Coy, et al, "Susceptibility of rare-earth-magnesium alloys to micro-galvanic corrosion," Corros. Sci., vol. 52, no.12, pp. 3896-3906, Dec. 2010.

[6] Z. Zhang, et al, "Characterization of phases in a Mg-6Gd-4Sm-0.4Zr (wt. \%) alloy during solution treatment," Mater. Charact.,vol. 60, no.6, pp.555-559, Jun. 2009

[7] P.J. Apps, et al, "Phase compositions in magnesiumrare earth alloys containing yttrium, gadolinium or dysprosium," Scripta Mater., vol. 48, no.5, pp.475-481, Mar. 2003.

[8] G. Ling, et al, "Corrosion Mechanisms of Magnesium Alloys," Adv. Eng. Mater., vol. 1, no.1, pp. 11-33, 1999.

[9] R. Zeng, et al, "Review of studies on corrosion of magnesium alloys,” T. Nonferr. Metal. Soc., vol. 16, no. 2, pp. 763-771,Jun. 2006.

[10] Y. Tamar and D. Mandler, "Corrosion inhibition of magnesium by combined zirconia silica sol-gel films," Electrochim. Acta, vol. 53, no.16, pp. 5118-5127, Jun. 2008.

[11] D.M. Liu, et al, "Water-based sol-gel synthesis of hydroxyapatite: process development," Biomaterials, vol. 22, no.13, pp. 1721-1730, Jul. 2001.

[12] D. Wang, and G. Bierwagen, "Sol-gel coatings on metals for corrosion protection," Prog. Org. Coat., vol. 64, no.4, pp.327-338, Mar. 2009.

[13] J.M. Guglielmi, "Sol-Gel coatings on metals," J. Sol-Gel Sci. Technol., no.8, pp.443-449, 1997.

[14] S. Zheng, et al, "Inorganic-organic sol gel hybrid coatings for corrosion protection of metals," J. Sol-Gel Sci. Technol., vol. 54, no. 2, pp.174-187, May. 2010.

[15] A.F. Galio, et al, "Inhibitor-doped sol-gel coatings for corrosion protection of magnesium alloy AZ31," Surf. Coat. Technol., vol. 204, no. 9-10, pp.1479-1486, Jan. 2010.

[16] Brusciotti, F., et al. Hybrid epoxy-silane coatings for improved corrosion protection of Mg alloy. Corros. Sci., vol. 67, pp. 82-90. Feb. 2013.

[17] López, A.J., et al. Sol-gel coatings of low sintering temperature for corrosion protection of ZE41 magnesium alloy. Surf. Coat. Tech,. vol. 205, no. 17-18, pp. 41834191, May. 2011.

[18] M. Damron, et al, "Inorganic/organic hybrid coatings for aircraft aluminum alloy substrates," Prog. Org. Coat., vol. 41, no. 4, pp.226-232, May. 2001.

[19] "Standard Practice for Microetching Metals and Alloys," Norma ASTM E407-07e1, Filadelfia (EE.UU.): American Society of Testing and Materials (ASTM), vol. 03.01, 1999
[20] G. Garcés, et al, "Microstructural and mechanical characterisation of WE54-SiC composites," Mater. Sci. Eng., A, vol. 527, no. 24-25, pp. 6511-6517, Sep. 2010.

[21] "Standard Practice for Conventions Applicable to Electrochemical Measurements in Corrosion Testing," Norma ASTM G3-89, Filadelfia (EE.UU.): American Society of Testing and Materials (ASTM), vol. 03.02, 1999. 